\title{
The Essential Oil Constituents and Antimicrobial Activity of Anthemis aciphylla BoIss. var. discoidea BoIss.
}

\author{
K. Hüsnü Can Baser, ${ }^{a}$ Betül Demirci, ${ }^{a}$ Gökalp Iscan, ${ }^{a}$ Toshihoro Hashimoto, ${ }^{b}$ Fatih Demirci, ${ }^{a}$ \\ Yoshiaki Noma, ${ }^{c}$ and Yoshinori AsAKAWA ${ }^{*, b}$ \\ ${ }^{a}$ Department of Pharmacognosy, Faculty of Pharmacy, Anadolu University; 26470-Eskişehir, Turkey: ${ }^{b}$ Faculty of \\ Pharmaceutical Sciences, Tokushima Bunri University; and ${ }^{c}$ Faculty of Domestic Sciences, Tokushima Bunri University; \\ 180 Yamashiro-cho, Tokushima 770-8055, Japan. $\quad$ Received July 15, 2005; accepted October 12, 2005
}

The essential oil of aerial parts, leaves and flowers of the endemic Anthemis aciphylla BoIss. var. discoidea BoIss. (Asteraceae) were obtained by hydrodistillation. The oils were analyzed both by GC and GC-MS on a polar column. The monoterpenes $\alpha$-pinene $(9-49 \%)$ and terpinen-4-ol $(22-32 \%)$ were characterized as the main constituents. An unknown component isolated from the essential oil was characterized by means of MS, HR-MS, FT-IR, 1D- and 2D-NMR techniques as isofaurinone (1). Furthermore, the biological activity of the essential oils was evaluated in various human pathogenic microorganisms using the broth microdilution method. Weak to moderate inhibitions $(0.06-1.0 \mathrm{mg} / \mathrm{ml})$ was observed.

Key words Anthemis aciphylla var. discoidea; Asteraceae; essential oil; isofaurinone; antimicrobial activity

The genus Anthemis L. (Asteraceae) is represented in the flora of Turkey by 81 taxa belonging to 51 species, 29 of which are endemic to Turkey. Anthemis aciphylla Boiss. var. discoidea Borss. is also one of the endemic species. ${ }^{1,2)} \mathrm{An}$ themis species are known to possess various biological activities and are commonly used in folk medicine. A. nobilis L., commonly known as Roman chamomile, is used like German chamomile (Matricaria chamomilla L.) and in Turkey, it is considered superior to German chamomile. Dried flowers of A. altissima L., A. hyalina DC., A. arvensis L., A. auriculata Borss., A. chia L., A. cotula L. and A. tinctoria L. are used as a replacement for German chamomile and as stimulant, emmenagogue, and carminative infusions. ${ }^{3)}$

A literature survey showed the presence of acetylenes, dehydromatricaria ester, thioenolether, and several sesquiterpenes which were isolated from the aerial parts and the roots of A. aciphylla var. discoidea. ${ }^{4)}$ To the best of our knowledge, this is the first report on the essential oil composition and biological activity of the different parts of this species.

\section{Results and Discussion}

Dried aerial parts, leaves and flowers were subjected to hydrodistillation and the obtained essential oils were analysed by GC and GC/MS. Overall 66, 77 and 62 compounds were characterized in these oils of $A$. aciphylla var. discoides, respectively. The main components were identified as $\alpha$-pinene $(49.4,9.4,39.0 \%)$ and terpinen-4-ol $(21.8,24.3,32.1 \%)$, respectively. Non-oxygenated monoterpenes were the predominant group of constituents in the essential oil of aerial parts and flowers while oxygenated monoterpenes were the predominant group of constituents in the leaves. The cumulative results are given in Table 1.

Interestingly, an unknown constituent with $222\left[\mathrm{M}^{+}\right]$was detected in the GC-MS analysis. The amount of this compound was $5.2 \%$ in the leaf oil which enabled its isolation by silica gel column chromatography to afford a new sesquiterpene, isofaurinone (1) related to faurinone (2) from Valeriana offcinalis first reported by Hikino et al., ${ }^{5)}$ which is known to be a rearranged derivative of eudesmane. Some stereochemical variations have been reported. ${ }^{5,6)}$ Compound
1 was prepared from (-)-carvomenthone by a multi-step synthetic procedure. $\left.{ }^{7}\right)$ However, a new structure (3) for faurinone was proposed with careful analysis of ${ }^{1} \mathrm{H}$ - and ${ }^{13} \mathrm{C}$ NMR spectra by Bos et al. ${ }^{8}$ However, to the best of our knowledge, the stereochemistry indicates it is a new essential oil derivative from a natural source. Influenced by the biogenetical evidence that the C-7 isopropyl group is in the $\beta$ orientation, circular dichroism studies along low resolution NMR was employed formerly to deduct the absolute chemistry of the eudesmane sesquiterpenes. ${ }^{5}$ In the present work, structure elucidation of $\mathbf{1}$ was carried out using spectral techniques. A new sesquiterpene (1) was obtained as a colorless oil, whose molecular formula, $\mathrm{C}_{15} \mathrm{H}_{26} \mathrm{O}$ was established by high resolution electron impact mass spectroscopy (HR-EIMS) $\left([\mathrm{M}]^{+} m / z\right.$ 222.1977). The FT-IR spectrum of 1 indicated the presence of a carbonyl $\left(1716 \mathrm{~cm}^{-1}\right)$ group, while the ${ }^{13} \mathrm{C}$-NMR spectrum of 1 showed 15 carbon signals. As indicated in Table 3 , the ${ }^{1} \mathrm{H}$ - and ${ }^{13} \mathrm{C}$-NMR spectra of $\mathbf{1}$ showed the presence of two secondary methyl groups $\left[\delta_{\mathrm{H}}\right.$ $0.83(6 \mathrm{H}, \mathrm{d}, J=6.8 \mathrm{~Hz}, \mathrm{H}-12,13) ; \delta_{\mathrm{C}} 19.5(\mathrm{q}), 19.7$ $(\mathrm{q})]$, one tertiary methyl $\left[\delta_{\mathrm{H}} 0.92(3 \mathrm{H}, \mathrm{s}) ; \delta_{\mathrm{C}} 25.2(\mathrm{q})\right]$ and one acetyl $\left[\delta_{\mathrm{H}} 2.15(3 \mathrm{H}, \mathrm{s}) ; \delta_{\mathrm{C}} 28.2(\mathrm{q}), 212.4(\mathrm{~s})\right]$. The ${ }^{1} \mathrm{H}-$ NMR spectral data of isofaurinone (1) were similar to those of faurinone (2) except for the chemical shifts of one secondary methyl $\left[\delta_{\mathrm{H}} 0.74(3 \mathrm{H}, \mathrm{d}, J=5.0 \mathrm{~Hz}, \mathrm{H}-12\right.$ or $\left.\mathrm{H}-13)\right]$ and one tertiary methyl group $\left[\delta_{\mathrm{H}} 1.03(3 \mathrm{H}, \mathrm{s}, \mathrm{H}-14)\right]$. Compound 1 showed the correlations between (i) $\mathrm{H}-3 / \mathrm{C}-1$ and $\mathrm{C}$ 4; (ii) $\mathrm{H}-6 / \mathrm{C}-4$ and $\mathrm{C}-8$ (iii) $\mathrm{H}-12 / \mathrm{C}-7, \mathrm{C}-11$ and $\mathrm{C}-13$; (iv) $\mathrm{H}-13 / \mathrm{C}-7, \mathrm{C}-11$ and $\mathrm{C}-12$; (iv) $\mathrm{H}-15 / \mathrm{C}-4$ and $\mathrm{C}-5$ in the HMBC spectrum (Fig. 1), and the NOEs between (i) H-7 and $\mathrm{H}-9$; (ii) $\mathrm{H}-12 / \mathrm{H}-8 \beta$ and $\mathrm{H}-11$; (iii) $\mathrm{H}-13 / \mathrm{H}-6 \beta$ and $\mathrm{H}-11$; (iv) $\mathrm{H}-14 / \mathrm{H}-3 \beta$ and $\mathrm{H}-9 \beta$; and (v) $\mathrm{H}-15 / \mathrm{H}-3 \alpha$ and $\mathrm{H}-6 \alpha$ in the NOESY spectrum (as seen in Fig. 1). From the above described spectral evidence, the relative structure of the new compound (1) was named isofaurinone, which was deduced to be a regioisomer of the acetyl group of faurinone (2) at C5 . The sequiterpene-skeleton of $\mathbf{1}$ was very rare. Only $3 \alpha, 11$ dihydroxy-isoiphionan-4-one-[ $\alpha$-xylopyranoside] from Iphiona scabra is known in the natural kingdom. ${ }^{9)}$

Furthermore, the essential oils were subjected to an an- 
Table 1. The Composition of the Essential Oils of Anthemis aciphylla var. discoidea

\begin{tabular}{|c|c|c|c|c|c|}
\hline No. & Compound & RRI & $\mathrm{A}(\%)$ & B $(\%)$ & $\mathrm{C}(\%)$ \\
\hline 1 & $\alpha$-Pinene & 1032 & 49.4 & 9.4 & 39.0 \\
\hline 2 & Hexanal & 1093 & - & - & 0.1 \\
\hline 3 & $\beta$-Pinene & 1118 & 4.2 & 1.6 & 4.3 \\
\hline 4 & Sabinene & 1132 & 0.5 & 0.1 & 0.5 \\
\hline 5 & Myrcene & 1174 & 0.6 & 0.1 & 0.6 \\
\hline 6 & $\alpha$-Terpinene & 1188 & 0.3 & $\operatorname{tr}$ & 0.3 \\
\hline 7 & Limonene & 1203 & 1.5 & 2.1 & 3.4 \\
\hline 8 & 1,8-Cineole & 1213 & 0.1 & 0.1 & 0.1 \\
\hline 9 & $\beta$-Phellandrene & 1218 & 0.1 & - & 0.1 \\
\hline 10 & Amyl furan (=2-Pentyl furan) & 1244 & 0.1 & $\operatorname{tr}$ & 0.1 \\
\hline 11 & $(Z)-\beta$-Ocimene & 1246 & 0.1 & - & 0.1 \\
\hline 12 & $\gamma$-Terpinene & 1255 & 0.6 & 0.1 & 0.4 \\
\hline 13 & $(E)$ - $\beta$-Ocimene & 1266 & 0.5 & $\operatorname{tr}$ & 0.5 \\
\hline 14 & $p$-Cymene & 1280 & 0.5 & 0.5 & 0.4 \\
\hline 15 & Terpinolene & 1290 & 0.3 & 0.1 & 0.2 \\
\hline 16 & Octanal & 1296 & 0.1 & 0.1 & - \\
\hline 17 & Nonanal & 1400 & 0.4 & 0.4 & 0.4 \\
\hline 18 & $\gamma$-Campholene aldehyde & 1439 & - & 0.1 & - \\
\hline 19 & (E)-2-Octenal & 1441 & 0.2 & 0.1 & 0.2 \\
\hline 20 & $\alpha$-Copaene & 1497 & 0.6 & 0.9 & 0.5 \\
\hline 21 & $\alpha$-Campholene aldehyde & 1499 & - & 0.8 & - \\
\hline 22 & Decanal & 1506 & - & 0.4 & - \\
\hline 23 & Chrysanthenone & 1522 & - & 0.1 & - \\
\hline 24 & $\beta$-Bourbonene & 1535 & 0.2 & 0.8 & 0.3 \\
\hline 25 & (E)-2-Nonenal & 1548 & 0.1 & 0.2 & 0.2 \\
\hline 26 & Linalool & 1553 & 0.1 & 0.2 & 0.1 \\
\hline 27 & Octanol & 1562 & - & 0.1 & 0.1 \\
\hline 28 & trans-p-Menth-2-en-1-ol & 1571 & 0.1 & 0.2 & 0.1 \\
\hline 29 & Pinocarvone & 1586 & - & 0.5 & - \\
\hline 30 & Terpinen-4-ol & 1611 & 21.8 & 24.3 & 32.1 \\
\hline 31 & Octyl 2-methyl butyrate & 1634 & 0.1 & - & - \\
\hline 32 & cis-p-Menth-2-en-1-ol & 1638 & - & 0.2 & 0.1 \\
\hline 33 & Myrtenal & 1648 & - & 0.5 & - \\
\hline 34 & Octyl 3-methyl butyrate (=Octyl isovalerate) & 1654 & 0.2 & - & - \\
\hline 35 & (E)-2-Decenal & 1655 & $\operatorname{tr}$ & 0.7 & 0.2 \\
\hline 36 & cis-Verbenol & 1663 & $\operatorname{tr}$ & 0.7 & - \\
\hline 37 & Nonanol & 1664 & $\operatorname{tr}$ & - & - \\
\hline 38 & $(Z)-\beta$-Farnesene & 1668 & 0.1 & - & - \\
\hline 39 & trans-Pinocarveol & 1670 & 0.2 & 1.1 & 0.2 \\
\hline 40 & $p$-Mentha-1,5-dien-8-ol & 1674 & - & 0.2 & - \\
\hline 41 & trans-Verbenol & 1683 & 0.3 & 2.7 & 0.3 \\
\hline 42 & Salicylaldehyde & 1703 & - & - & 0.1 \\
\hline 43 & $\gamma$-Curcumene & 1704 & 0.3 & 0.4 & 0.5 \\
\hline 44 & $\alpha$-Terpineol & 1706 & 0.2 & 0.5 & 0.4 \\
\hline 45 & Borneol & 1719 & - & 0.3 & - \\
\hline 46 & $\alpha$-Zingiberene & 1726 & 0.2 & - & 0.4 \\
\hline 47 & Germacrene D & 1726 & 1.0 & 0.9 & 1.2 \\
\hline 48 & $(Z, E)$ - $\alpha$-Farnesene & 1737 & - & 0.1 & - \\
\hline 49 & $\beta$-Bisabolene & 1741 & 0.3 & 0.3 & 0.4 \\
\hline 50 & Carvone & 1751 & - & 0.4 & - \\
\hline 51 & Bicyclogermacrene & 1755 & 0.1 & - & - \\
\hline 52 & cis-Piperitol & 1758 & - & 0.1 & - \\
\hline 53 & (E)-2-undecenal & 1764 & 0.1 & 0.4 & - \\
\hline 54 & Decanol & 1766 & - & - & 0.1 \\
\hline 55 & $\delta$-Cadinene & 1773 & 0.3 & 0.3 & 0.4 \\
\hline 56 & $\gamma$-Cadinene & 1776 & 0.1 & 0.3 & 0.1 \\
\hline 57 & $(E, Z)$-2,4-Decadienal & 1779 & 0.1 & 0.1 & 0.1 \\
\hline 58 & ar-Curcumene & 1786 & $\operatorname{tr}$ & 0.1 & 0.1 \\
\hline 59 & Myrtenol & 1804 & 0.1 & 0.8 & 0.1 \\
\hline 60 & $(E, E)$-2,4-Decadienal & 1827 & 0.2 & 0.5 & 0.2 \\
\hline 61 & trans-Carveol & 1845 & - & 0.7 & 0.2 \\
\hline 62 & $p$-Cymen-8-ol & 1864 & - & 0.4 & - \\
\hline 63 & (E)-Geranyl acetone & 1868 & - & $\operatorname{tr}$ & - \\
\hline 64 & cis-Carveol & 1882 & - & 0.1 & - \\
\hline 65 & Geranyl isovalerate & 1893 & - & 0.1 & - \\
\hline 66 & Dodecyl acetate & 1893 & 0.1 & - & - \\
\hline
\end{tabular}


Table 1. continued

\begin{tabular}{|c|c|c|c|c|c|}
\hline No & Compound & RRI & A $(\%)$ & $\mathrm{B}(\%)$ & $\mathrm{C}(\%)$ \\
\hline 67 & Isofaurinone (=Compound $\mathbf{1})$ & 1904 & 0.5 & 5.2 & 0.5 \\
\hline 68 & Caryophyllene oxide & 2008 & 0.3 & 1.1 & 0.2 \\
\hline 69 & Salvial-4(14)-en-1-one & 2037 & 0.1 & 0.6 & - \\
\hline 70 & Pentadecanal & 2041 & 0.1 & $\operatorname{tr}$ & 0.1 \\
\hline 71 & Ledol & 2057 & - & 0.4 & - \\
\hline 72 & Octanoic acid & 2084 & - & 0.2 & 0.1 \\
\hline 73 & Hexahydrofarnesyl acetone & 2131 & 0.3 & 1.0 & 0.3 \\
\hline 74 & Spathulenol & 2144 & 0.3 & 0.8 & 0.3 \\
\hline 75 & (Z)-3-Hexen-1-yl benzoate & 2148 & 0.1 & - & - \\
\hline 76 & Tetradecanol & 2179 & - & 0.5 & - \\
\hline 77 & Nonanoic acid & 2192 & 0.1 & 0.9 & 0.2 \\
\hline 78 & Thymol & 2198 & 0.2 & 0.9 & 0.1 \\
\hline 79 & 4-Vinyl guaiacol & 2218 & $\operatorname{tr}$ & - & $\operatorname{tr}$ \\
\hline 80 & Carvacrol & 2239 & 0.1 & 0.1 & $\operatorname{tr}$ \\
\hline 81 & $\alpha$-Cadinol & 2255 & 0.1 & - & 0.3 \\
\hline 82 & Selin-11-en- $4 \alpha$-ol & 2273 & - & 0.2 & - \\
\hline 83 & Decanoic acid & 2298 & 0.4 & 1.5 & 0.6 \\
\hline 84 & Tricosane & 2300 & 0.6 & 0.6 & 0.2 \\
\hline 85 & $\gamma$-Dodecalactone & 2396 & - & 0.3 & - \\
\hline 86 & Pentacosane & 2500 & $\operatorname{tr}$ & 0.2 & $\operatorname{tr}$ \\
\hline 87 & Dodecanoic acid & 2503 & 0.5 & 1.7 & 0.5 \\
\hline 88 & 1-Octadecanol & 2607 & 0.1 & 0.5 & - \\
\hline 89 & Tetradecanoic acid & 2670 & 0.2 & 0.9 & 0.1 \\
\hline 90 & Heptacosane & 2700 & 0.2 & 0.1 & 0.1 \\
\hline 91 & Nonacosane & 2900 & 0.2 & 0.5 & 0.1 \\
\hline \multirow[t]{8}{*}{92} & Hexadecanoic acid & 2931 & 0.3 & 1.3 & 0.2 \\
\hline & \multicolumn{2}{|l|}{ Monoterpenes } & 58.6 & 14.0 & 49.8 \\
\hline & \multicolumn{2}{|l|}{ Oxygenated monoterpenes } & 23.2 & 35.6 & 33.8 \\
\hline & \multicolumn{2}{|l|}{ Sesquiterpenes } & 3.2 & 4.6 & 3.9 \\
\hline & \multicolumn{2}{|l|}{ Oxygenated sesquiterpenes } & 1.3 & 8.3 & 1.3 \\
\hline & \multicolumn{2}{|l|}{ Others } & 4.8 & 13.2 & 4.3 \\
\hline & \multicolumn{2}{|l|}{ Identified compounds } & 66 & 77 & 62 \\
\hline & \multicolumn{2}{|l|}{ Total } & 91.1 & 75.7 & 93.1 \\
\hline
\end{tabular}

A: aerial parts, B: leaves, C: flowers. RRI: Relative retention indices calculated against $n$-alkanes. \%: calculated from FID data. tr: trace $(<0.1 \%)$.

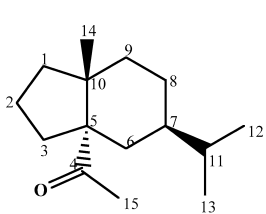

1

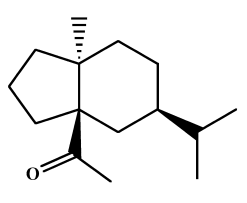

2

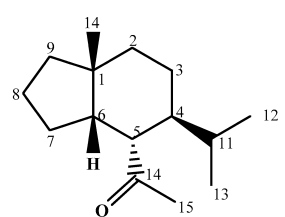

3

Fig. 1. Chemical Structures of Isofaurinone (1) and Faurinone $(\mathbf{2}, \mathbf{3})$

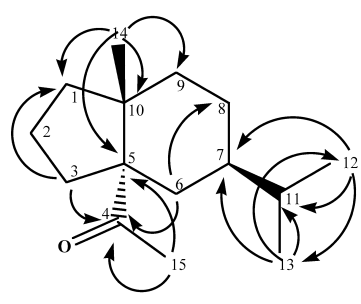

Important $\mathrm{HMBC}$ correlations

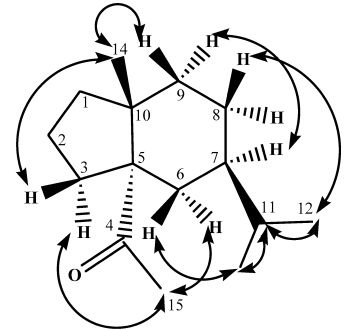

Important NOESY correlations

Fig. 2. $\mathrm{HMBC}$ and NOESY Spectra of Isofaurinone (1) in $\mathrm{CDCl}_{3}$

timicrobial bioassay $^{8)}$ for the determination of the minimal inhibitory concentrations (MIC). The results are given in Table 3. As an overall result, the essential oils showed weak to moderate inhibitory effects on the microorganisms used. Almost all of the essential oils tested were weaker in inhibition when compared to the standard antimicrobial agents chloramphenicol and ketoconazole at the same tested con-

Table 2. ${ }^{1} \mathrm{H}$ - and ${ }^{13} \mathrm{C}-\mathrm{NMR}$ Data of Compound $\mathbf{1}^{a)}$

\begin{tabular}{|c|c|c|}
\hline Position no. & ${ }^{1} \mathrm{H}(\delta)$ & ${ }^{13} \mathrm{C}(\delta)$ \\
\hline $1 \alpha$ & $2.00(\mathrm{~m})$ & $34.9(\mathrm{t})$ \\
\hline $1 \beta$ & $1.14(\mathrm{~m})$ & \\
\hline 2 & $1.72(\mathrm{~m})$ & $18.0(\mathrm{t})$ \\
\hline $3 \alpha$ & $1.66(\mathrm{~m})$ & $34.8(\mathrm{t})$ \\
\hline $3 \beta$ & $2.18(\mathrm{~m})$ & \\
\hline 4 & & $212.4(\mathrm{~s})$ \\
\hline 5 & & $59.9(\mathrm{~s})$ \\
\hline $6 \alpha$ & 1.47 (br d, $J=11.8)$ & $33.4(\mathrm{t})$ \\
\hline $6 \beta$ & $1.11(\mathrm{dd}, J=5.5,11.8)$ & \\
\hline 7 & $1.14(\mathrm{~m})$ & $38.8(d)$ \\
\hline $8 \alpha$ & $1.52(\mathrm{~m})$ & $24.8(\mathrm{t})$ \\
\hline $8 \beta$ & $1.15(\mathrm{~m})$ & \\
\hline $9 \alpha$ & $1.42(\mathrm{~m})$ & $35.2(\mathrm{t})$ \\
\hline $9 \beta$ & $1.88(\mathrm{~m})$ & \\
\hline 10 & & $42.7(\mathrm{~s})$ \\
\hline 11 & $1.34(\mathrm{~m})$ & $32.4(d)$ \\
\hline 12 & $0.83(\mathrm{~d}, J=6.8)$ & $19.5(q)^{b)}$ \\
\hline 13 & $0.83(\mathrm{~d}, J=6.8)$ & $19.7(\mathrm{q})^{b)}$ \\
\hline 14 & $0.92(\mathrm{~s})$ & $25.2(q)$ \\
\hline 15 & $2.15(\mathrm{~s})$ & $28.2(q)$ \\
\hline
\end{tabular}

a) Chemical shifts from TMS (multiplicity, $J$ in $\mathrm{Hz}$ ) in $\mathrm{CDCl}_{3}$ (Assignments from ${ }^{1} \mathrm{H}-{ }^{1} \mathrm{H}$ COSY, NOESY, HMQC and HMBC spectra, see Fig. 1). b) May be interchanged in each vertical column.

centration. Staphylococcus epidermidis was most strongly inhibited by Anthemis oils with MIC values of $0.125,0.06$ and $0.25 \mathrm{mg} / \mathrm{ml}$, respectively. 
Table 3. Antimicrobial Activity (MIC, $\mathrm{mg} / \mathrm{ml}$ ) of Anthemis aciphylla var. discoidea Oils

\begin{tabular}{lllll}
\hline \hline \multicolumn{1}{c}{ Microorganisms } & $\mathrm{A}$ & $\mathrm{B}$ & $\mathrm{C}$ & $\mathrm{ST}$ \\
\hline Escherichia coli & 1.0 & 0.5 & 1.0 & 0.06 \\
Staphylococcus aureus & 0.5 & 0.5 & 1.0 & 0.007 \\
Pseudomonas aeruginosa & 1.0 & 0.5 & 0.25 & 0.25 \\
Enterobacter aerogenes & 1.0 & 1.0 & 0.5 & 0.125 \\
Staphylococcus epidermidis & 0.125 & 0.06 & 0.25 & 0.007 \\
Salmonella typhimurium & 1.0 & 0.5 & 1.0 & 0.06 \\
Candida albicans & 0.5 & 0.25 & 0.5 & $0.06^{a)}$ \\
\hline
\end{tabular}

A: aerial parts, B: leaves, C: flowers, ST: chloramphenicol. a) Ketaconazole for the yeast.

\section{Experimental}

General Experimental Procedures IR was measured using a JASCOFT-IR-5300 apparatus. ${ }^{1} \mathrm{H}$ - and ${ }^{13} \mathrm{C}-\mathrm{NMR}$ spectra were recorded on Varian UNITY 600 system at 600 and $150 \mathrm{MHz}$, respectively. Chemical shifts are given relative to tetramethylsilane (TMS) at $0.0 \mathrm{ppm}$ as internal standard in $\mathrm{CDCl}_{3}$. 1D- and 2D-NMR data were also obtained using the same system, and the spectra were measured and reported in ppm. HR-MS analysis was conducted using a JEOL JMS-AX-500 system. All chemicals were obtained from Sigma/Aldrich.

Plant Material The plant material was collected in Eskişehir: Bozdağ, Turkey, in July 2001. Voucher specimens are kept at the Herbarium of the Faculty of Pharmacy of Anadolu University in Eskişehir, Turkey (ESSE 13180).

Hydrodistillation Air dried aerial parts, leaves and flowers of plant material was hydrodistilled for $3 \mathrm{~h}$ using a Clevenger-type apparatus to yield essential oil $(0.09 \%, 0.11 \%, 0.35 \%$, respectively).

Gas Chromatography (GC) The oils were analyzed by GC using a Hewlett Packard 6890 system. An HP-Innowax FSC column $(60 \mathrm{~m} \times 0.25 \mathrm{~mm}$ inner diameter, with $0.25 \mu \mathrm{m}$ film thickness) was used with nitrogen as carrier gas $(1 \mathrm{ml} / \mathrm{min})$. The oven temperature was kept at $60{ }^{\circ} \mathrm{C}$ for $10 \mathrm{~min}$ and programmed to $220^{\circ} \mathrm{C}$ at a rate of $4{ }^{\circ} \mathrm{C} / \mathrm{min}$, then kept constant at $220^{\circ} \mathrm{C}$ for $10 \mathrm{~min}$ and then programmed to $240^{\circ} \mathrm{C}$ at a rate of $1{ }^{\circ} \mathrm{C} / \mathrm{min}$. The injector temperature was set at $250^{\circ} \mathrm{C}$. The percentage compositions were obtained from electronic integration measurements using flame ionization detection (FID, $250^{\circ} \mathrm{C}$ ). $n$-Alkanes were used as reference points in the calculation of relative retention indices (RRI). Relative percentages of the characterized components were as cited in Table 1.

Gas Chromatography-Mass Spectrometry (GC-MS) The essential oils were analysed by gas chromatography/mass spectrometry using a Hewlett-Packard GCD system. An Innowax FSC column $(60 \mathrm{~m} \times 0.25 \mathrm{~mm}$ inner diameter, $0.25 \mu \mathrm{m}$ film thickness) was used with helium as carrier gas. The GC oven temperature was as above. Split flow was adjusted at $50 \mathrm{ml} / \mathrm{min}$. The injector temperature was $250^{\circ} \mathrm{C}$. MS were taken at $70 \mathrm{eV}$. Mass range was from $m / z 35$ to 425 . A library search was carried out using both the "Wiley GC/MS Library" and the in-house "Baser Library of Essential Oil Constituents".
Isolation of Isofaurinone (1) The compound was isolated by microcolumn chromatography. Silica Gel $60 \mathrm{G}$ ( $c a .1 \mathrm{~g}$, Merck 7734) was used as the packing material and was added to a Pasteur pipette with wet $n$-hexane. $n$-Hexane in diethylether $(100 \% \rightarrow 0 \%)$ was used as the eluent in the gradient system. Essential oil $(15 \mathrm{mg})$ was applied and eluted with $n$-hexane: diethylether (99:1) to yield $\mathbf{1}(2.1 \mathrm{mg})$ as a colorless oily material.

FT-IR $v_{\max }\left(\mathrm{cm}^{-1}\right): 2956,2870,1716(\mathrm{C}=\mathrm{O}), 1463,1385,879$; The ${ }^{1} \mathrm{H}-$ and ${ }^{13} \mathrm{C}$-NMR data are given in Table 2; HR-EI-MS $222.1977[\mathrm{M}]^{+} \mathrm{m} / \mathrm{z}$ : (Calcd for $\left.\mathrm{C}_{15} \mathrm{H}_{26} \mathrm{O} 222.1984\right)$, EI-MS $m / z$ (rel. int.)=222 (3) $\left[\mathrm{M}^{+}\right], 207$ (1), 179 (6), 161 (1), 149 (1), 137 (2), 125 (100), 109 (9), 95 (11), 81 (10), 69 (7), 55 (7), 43 (33).

Antimicrobial Assay Escherichia coli (ATCC 25922), Staphylococcus aureus (ATCC 6538), Pseudomonas aeruginosa (ATCC 27853), Staphylococcus epidermidis (ATCC 12228), Enterobacter aerogenes (NRRL 3567), Salmonella typhimurium (NRRL 4420), and Candida albicans (clinical isolate, Osmangazi University, Faculty of Medicine, Eskisehir, Turkey) were used as test microorganisms. The bacteria and yeast were refreshed in Mueller Hinton Broth (Merck, Germany) at $35-37^{\circ} \mathrm{C}$ prior to use.

A micro-dilution broth susceptibility assay was performed according to our previously described method. ${ }^{10)}$ Stock solutions of the essential oils were prepared in diluted dimethylsulfoxide (Carlo-Erba, France). Dilution series were prepared from $2 \mathrm{mg} / \mathrm{ml}$ to $0.001 \mathrm{mg} / \mathrm{ml}$ in sterile distilled water in micro-test tubes from where they were transferred to 96 -well microtiter plates. All microorganisms grown overnight in double strength Mueller Hinton Broth were standardized to approx. $10^{8} \mathrm{CFU} / \mathrm{ml}$ (using McFarland No: 0.5 ). The last row containing only the serial dilutions of sample without microorganism was used as a negative control. Sterile distilled water and medium served as a positive growth control. After incubation at $37^{\circ} \mathrm{C}$ for $18-24 \mathrm{~h}$, the minimal inhibitory concentrations (MIC) were determined (see Table 3). Chloramphenicol and ketoconazole (Sigma, Germany) were used as standard antimicrobial agents.

\section{References}

1) Davis P. H., "Flora of Turkey and the East Aegean Islands," Vol. 5, University Press, Edinburgh, 1975.

2) Güner A., Özhatay N., Ekim T., Baser K. H. C., "Flora of Turkey and the East Aegean Islands," Vol. 11, University Press, Edinburgh, 2000.

3) Baytop T., “Türkiye'de Bitkiler ile Tedavi, Geçmişte ve Bugün (Therapy with Medicinal Plants in Turkey, Past and Present),“ 2nd ed., Nobel Tip Kitabevleri, Istanbul, 1999.

4) Bohlmann F., Zdero, C., Chem Ber., 108, 1902-1910 (1975).

5) Hikino H., Hikino Y., Agatsuma K., Takemoto T., Chem. Pharm. Bull., 16, 1779-1783 (1968).

6) Hikino H., Kohama T., Takemoto T., Tetrahedron, 25, 1037-1045 (1969).

7) Burnell R. H., Leroux M., Synth. Commun., 13, 559-567 (1983).

8) Bos R., Hendriks H., Kloosterman J., Sipma G., Phytochemistry, 22, 1505-1506 (1983).

9) El-Ghazouly M. G., Ei-Sebakhy N. A., Seif El-Din A. A., Zdero C., Bohlmann F., Phytochemistry, 26, 439-443 (1987).

10) Iscan G., Kırımer N., Kurkcuoglu M., Baser K. H. C., Demirci F., J. Agric. Food Chem., 50, 3943-3946 (2002). 